\title{
Diversity and distribution of alpha satellite DNA in the genome of an Old World monkey: Cercopithecus solatus
}

\author{
Lauriane Cacheux ${ }^{1,2}$, Loïc Ponger ${ }^{1}$, Michèle Gerbault-Seureau², Florence Anne Richard ${ }^{2,3}$ and Christophe Escudé ${ }^{* *}$
}

\begin{abstract}
Background: Alpha satellite is the major repeated DNA element of primate centromeres. Evolution of these tandemly repeated sequences has led to the existence of numerous families of monomers exhibiting specific organizational patterns. The limited amount of information available in non-human primates is a restriction to the understanding of the evolutionary dynamics of alpha satellite DNA.

Results: We carried out the targeted high-throughput sequencing of alpha satellite monomers and dimers from the Cercopithecus solatus genome, an Old World monkey from the Cercopithecini tribe. Computational approaches were used to infer the existence of sequence families and to study how these families are organized with respect to each other. While previous studies had suggested that alpha satellites in Old World monkeys were poorly diversified, our analysis provides evidence for the existence of at least four distinct families of sequences within the studied species and of higher order organizational patterns. Fluorescence in situ hybridization using oligonucleotide probes that are able to target each family in a specific way showed that the different families had distinct distributions on chromosomes and were not homogeneously distributed between chromosomes.

Conclusions: Our new approach provides an unprecedented and comprehensive view of the diversity and organization of alpha satellites in a species outside the hominoid group. We consider these data with respect to previously known alpha satellite families and to potential mechanisms for satellite DNA evolution. Applying this approach to other species will open new perspectives regarding the integration of satellite DNA into comparative genomic and cytogenetic studies.
\end{abstract}

Keywords: Alpha satellite DNA, High-throughput sequencing, Cercopithecus solatus, Centromere genomics

\section{Background}

Centromeres are chromosomal regions that control chromosome segregation during cell division in eukaryotes, through kinetochore assembly and microtubule attachment. In almost all eukaryotes, the DNA underlying centromeres is made of large tracts of nearly identical tandem DNA repeats, known as satellite DNA [1-3]. The remarkable variation of satellite DNAs between species has been an enigma ever since their discovery and different important roles have been ascribed to these

\footnotetext{
* Correspondence: escude@mnhn.fr

'Département Régulations, Développement et Diversité Moléculaire, Structure et Instabilité des Génomes, INSERM U1154, CNRS UMR7196, Sorbonne Universités, Muséum national d'Histoire naturelle, Paris, France Full list of author information is available at the end of the article
}

sequences, from the imperative centromeric function in mitosis and meiosis to regulatory functions $[4,5]$.

Alpha satellite DNA is the most abundant satellite DNA in Primates and is found both at the site of centromere attachment and in neighboring heterochromatic regions, referred to as pericentromeric regions [6]. Alpha satellite DNA was originally isolated as a highly repetitive component of the Chlorocebus aethiops (also called African green monkey) genome [7]; homologous repeats were then described throughout the Primate order including apes, Old World and New World monkeys [8-10]. Alpha satellite DNA is made of tandemly repeated AT-rich monomers that are about $170 \mathrm{bp}$ in length and organized in head-to-tail orientation $[11,12]$. In the human genome, individual monomers share between 60 and 100\% sequence identity. The highly identical composition of successive repeats represents a 
technical challenge that has thwarted the complete assembly of centromeric DNA so far [13, 14]. Nevertheless, over the last 30 years, the systematic cloning and sequencing of many alpha satellite DNAs, combined with fluorescence in situ hybridization (FISH) experiments, has provided a thorough knowledge of alpha satellite DNA diversity and organization patterns in the human genome $[11,15,16]$ and, to a much lesser extent, in other primates [17-20].

In human, alpha satellite DNA has been shown to adopt two different organizations. In the so-called higher order repeat (HOR) organizational pattern, highly conserved repeat units (97-100\% sequence identity), each made of multiple $171 \mathrm{bp}$ monomers (up to more than 30 ), are found as an homogenized array that can extend over a multimegabasesized region [2, 13, 21-23]. This organization is typically found as very long arrays of alpha satellites at the centromere core of all human chromosomes. In pericentromeres, a second type of organization, called monomeric and involving arrays of single alpha satellite monomers which are less well conserved (70-90\% sequence identity), can coexist with HORs $[3,12]$. Sequence comparisons between human alpha satellite monomers have led to the description of up to seventeen different alpha satellite families, or monomer types $[19,21,24,25]$. Although the alpha satellite component of other primate genomes has been less intensively studied, there is some evidence for similar organizations in great apes, but additional families have been described and the composition of HORs as well as their chromosomal distribution differ when compared with human [12, 20, 26-28]. This implies that the structure and content of centromeric DNA can change in a few million years.

Although the mechanisms that gave rise to this diversity and organization are not precisely known, it is commonly accepted that the so-called concerted evolution of repetitive sequences is based on different mechanisms of non-reciprocal transfer occurring within or between chromosomes, such as unequal crossover, gene conversion, rolling circle replication and reinsertion, and transposon-mediated exchange [4, 29]. Such mechanisms enable series of amplification events, thereby creating new arrays of alpha satellites [12, 16, 3032]. The analysis of the different alpha satellite families found in assembled pericentromeric regions from specific human chromosomes revealed an age gradient of the families along each chromosome arm, which led to propose that during the course of evolution, new arrays of alpha satellites expand at the centromere core, thereby splitting and displacing older arrays distally onto each arm $[3,6,13,19,33]$.

Knowledge about alpha satellite DNA in species outside the hominoid group is very scarce, in particular in Old World monkeys, a clade that includes Colobinae, Papionini and Cercopithecini. The tribe Cercopithecini contains 35 species which have diversified within the last 10 million years $[34,35]$ and therefore represents a particularly interesting group for studying the evolution of satellite DNA.
Moreover, it has been reported that alpha satellite DNA is more abundant in some Cercopithecini species (up to 20\% of the genome of Chlorocebus aethiops) [36] than in great apes, where its contribution would reach only $3 \%$ of the genome [14]. Finally, enzymatic digestion of genomic DNA from various Old World monkey species can lead to a clear alpha satellite ladder pattern which is not observed when human or chimpanzee DNA is used, thereby pointing to different composition and organization of alpha satellite DNA in Old World monkeys [37].

In the present work, we have undertaken the targeted sequencing of the alpha satellite component of Cercopithecus solatus (or Sun-tailed monkey) as a representative species for the Cercopithecini [38]. Alpha satellite monomers and dimers were obtained by enzymatic digestion of genomic DNA and gel purification, then submitted to highthroughput sequencing. The obtained sequences were analyzed and classified into monomer families using computational approaches. Finally the genomic distribution of each family was studied by FISH using a collection of oligonucleotide probes that are able to distinguish different sequence variants. Our study provides evidence for the existence of two main families of monomers which differ in their chromosomal distribution, one being specifically distributed on centromeres while the other is found only at pericentromeric locations with a non-uniform distribution between chromosomes. Two other families are detected which are only found associated within a dimeric organization and are located for the greatest part on the $\mathrm{Y}$ chromosome and to a lesser extent on pericentromeres from other chromosomes. These data represent the most complete analysis of the diversity and distribution of alpha satellite sequences in an Old World monkey reported to date. Our experimental approach may be applied to other species, opening new perspectives regarding the integration of satellite DNA into comparative studies.

\section{Results \\ Retrieval of alpha satellite sequences from the Cercopithecus solatus genome}

Work conducted in the early 1980s had shown that enzymatic digestion of genomic DNA from Old World monkeys with several restriction enzymes resulted in a migration profile that was characteristic for alpha satellite DNA, i.e. with bands corresponding to one and multiple repeat units of about $\mathrm{n} \times 170 \mathrm{bp}$ in length $[8,39]$. In silico analysis of several sequences isolated from Chlorocebus aethiops led us to select the $\mathrm{XmnI}$ restriction endonuclease as a candidate that should cleave a majority of monomers. Experimental digestion of Cercopithecus solatus genomic DNA with this enzyme revealed the expected banding pattern (Additional file 1: Figure S1). We therefore decided to extract DNA from two bands corresponding to monomers and dimers of alpha satellites from an agarose gel and 
implemented high throughput sequencing on an Ion Torrent sequencing platform providing reads up to $400 \mathrm{nu}$ cleotide in length (see Methods).

204,990 and 353,683 raw sequences were obtained for the monomer and dimer samples, respectively. Four in silico filters were applied successively to both datasets: a quality filter keeping sequences with a Phred quality score superior to 25; an extremity filter keeping sequences with the XmnI restriction site at both ends; a length filter keeping sequences within the range 162-182 bp for monomers and 324-364 bp for dimers, and an alpha satellite filter keeping sequences similar to an alpha satellite reference sequence (see Methods). The number of sequences that remained after each filter is reported on Additional file 2: Table S1. A total of 100,713 sequences fitting with all the criteria was obtained from the monomer sample and represents what we call from now on the monomer dataset. For the dimer sample, only 3,568 were obtained, they represent the dimer dataset. The drastic reduction observed within the dimer dataset was mostly the consequence of the length filter and may reflect an intrinsic limitation of the sequencing technology, unable to obtain long reads when template sequences are made of two successive highly identical sequences. These sequences were nevertheless included for further analysis as they provided an additional source of information (see below).

\section{Characterization of alpha satellite diversity in the monomer dataset}

A principal component analysis (PCA) using the 5-mer nucleotide composition of DNA sequences was applied to the monomer dataset in order to compare these sequences and identify putative groups without direct alignment. Visualization of sequences into the plane formed by the two first components of the PCA revealed two main groups of alpha satellite monomers, as shown by the distribution of points on Fig. 1a. Monomers were classified into each group by using a hierarchical clustering analysis (HCA) based on a subset of sequences followed by a linear discriminant analysis (LDA) to extend the classification to all the sequences (see colors on Fig. 1a). The most important group, called $\mathrm{C} 1$ and shown in purple, contained $82 \%$ of the sequences and the other group, called $\mathrm{C} 2$ and shown in pastel green, contained the remaining $18 \%$. To address the quality of this classification, 500 sequences were then randomly selected within the complete monomer dataset and were used to generate a phylogenetic tree where branches were colored according to the monomer classification (Fig. 1b). The disposition of sequences from the $\mathrm{C} 1$ and $\mathrm{C} 2$ groups on this tree provided a further support to our classification into two groups. Moreover, this tree showed a higher degree of divergence between $\mathrm{C} 2$ sequences compared to $\mathrm{C} 1$ sequences. Actually, the comparison of a subset of 500 randomly selected sequences within each group showed that the average sequence identity inside $\mathrm{C} 1$ was $95 \%$, whereas the average sequence identity inside $\mathrm{C} 2$ was only $85 \%$. The consensus sequences of $\mathrm{C} 1$ and C2 were $172 \mathrm{bp}$ in length, and differed from each other by a total of 9 positions (Fig. 2). Finally, monomers were searched for the presence of CENP-B and pJalpha boxes [40]. A pJalpha box was present in the

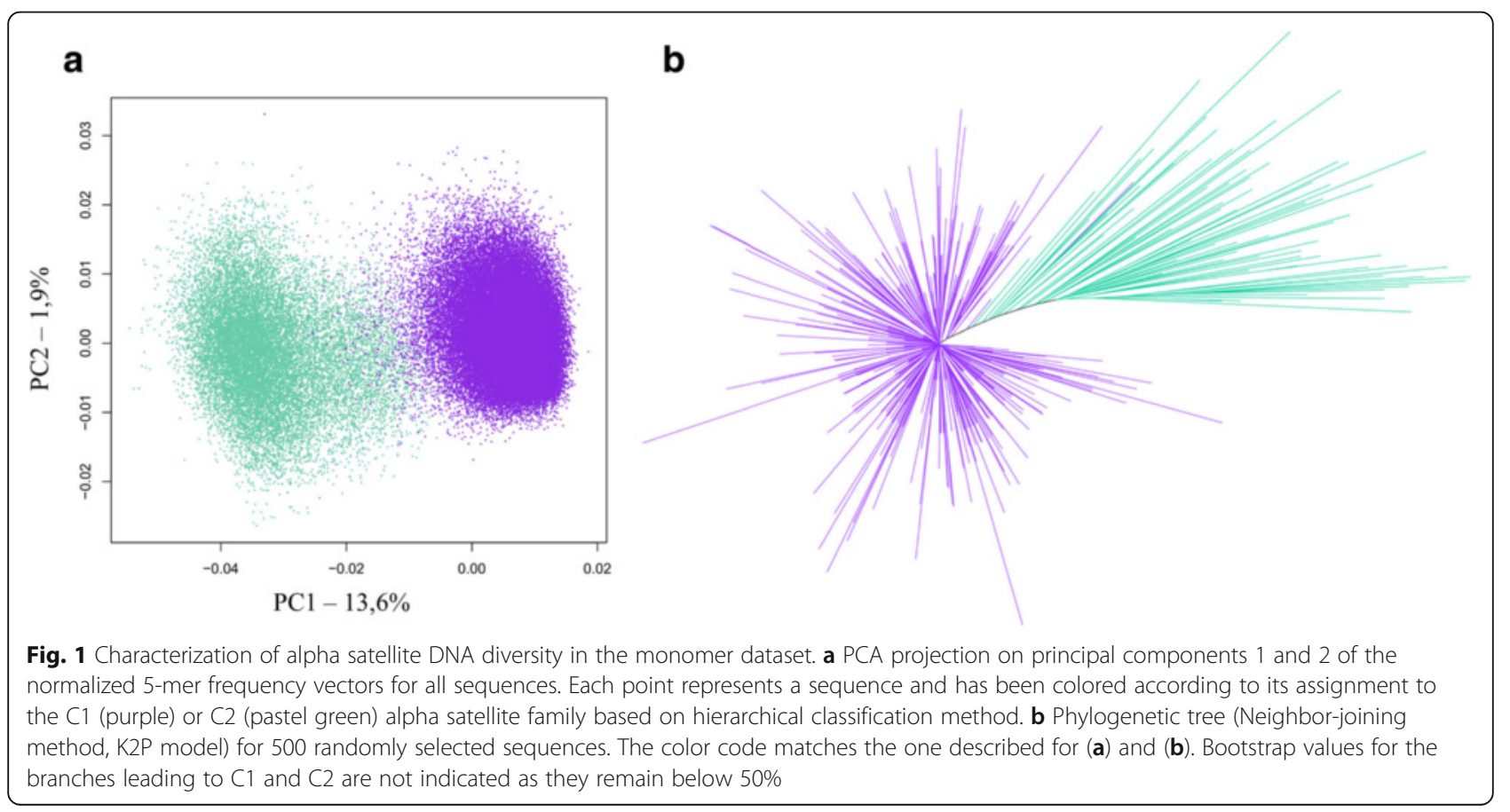




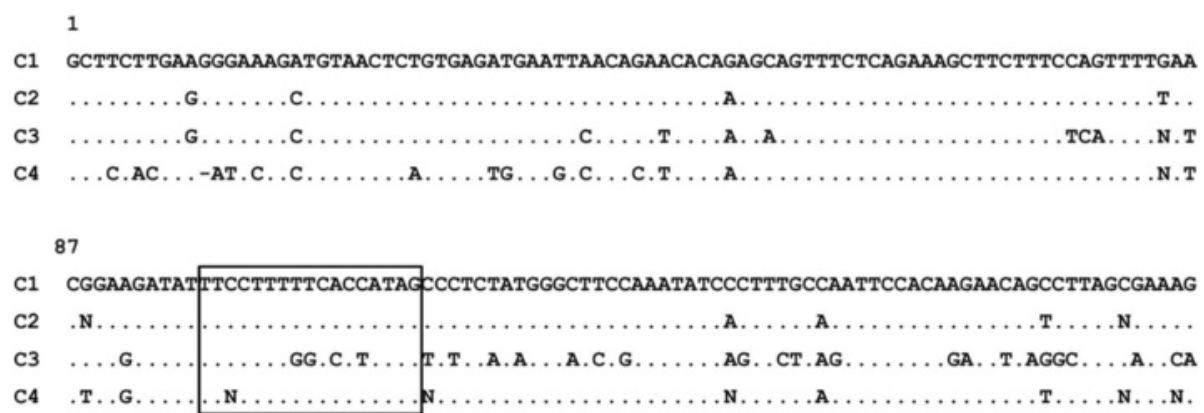

Fig. 2 Consensus sequences of the alpha satellite families identified in the Cercopithecus solatus genome. The consensus sequences were determined following the alignment of 500 randomly selected sequences for the C1 and C2 families, and the alignment of the available 109 and 112 sequences for the C3 and C4 families respectively. Each position was considered unambiguous if more than 60\% of monomers had the same nucleotide at this position. A point at a position replaces a nucleotide identical to the nucleotide at the homologous position in the C1 consensus. The box shows the fixation site of the pJalpha protein, which is absent from the C3 consensus

consensus of $\mathrm{C} 1$ and $\mathrm{C} 2$ and was found in $95 \%$ of $\mathrm{C} 1$ sequences and $85 \%$ of $\mathrm{C} 2$ sequences, whereas a CENPB box was only found in $0.05 \%$ and $0.04 \%$ of these sequences, respectively.

In order to further characterize the sequence diversity within the monomer dataset, we searched for the presence of identical sequences and noticed that while the sequences within the $\mathrm{C} 2$ group were all unique, numerous identical sequences could be found within the $\mathrm{C} 1$ group. A total of 4,850 sequences were repeated at least twice, representing a total of 20,248 reads in our dataset. Among those sequences, 20 were repeated more than 40 times and one 2,678 times. We decided to investigate further the 20 most abundant sequences. The most abundant sequence was exactly the consensus sequence of the $\mathrm{C} 1$ group, while all the others corresponded to this sequence with single nucleotide variations and/or deletions, as indicated on Table 1 . The absence of repeated sequences in the $\mathrm{C} 2$ group let us hypothesize, by contrast, that the different repeats observed in the $\mathrm{C} 1$ group may directly reflect the presence of strictly identical sequences in the Cercopithecus solatus genome. As Ion Torrent sequencing has been reported to give rise to sequencing errors, we decided to search if the identical sequences were obtained from reads collected in both orientations. We found that five out of the 20 sequences were associated with a strong bias for read orientation (Table 1). Within these five sequences, the three more abundant $(2,3,8)$ represented deletions within a homopolymer tract, while the two others $(15,20)$ corresponded to the combination of the two most abundant deletions (found in 2 and 3) with the most abundant single nucleotide variation (found in 4). Deletions within homopolymer tracts have already been shown to be inherent to the Ion torrent Technology [41] and the orientation bias we observed let us conclude that sequences displaying these deletions were non-relevant artifacts. On the contrary, all other sequences observed in high copy number, which were all obtained in both sequencing orientations, would correspond to sequence variants that are present with a high abundance in the Cercopithecus solatus genome.

Table 1 Analysis of alpha satellite sequences found in high copy number in the monomer dataset

\begin{tabular}{llll}
\hline Id & Sequence & Number & Forward (\%) \\
\hline 1 & Consensus & 2678 & 46 \\
2 & C114Del & 486 & $1^{*}$ \\
3 & T101Del & 357 & $99^{*}$ \\
4 & T39G & 242 & 46 \\
5 & A40C & 101 & 56 \\
6 & T121A & 92 & 41 \\
7 & T74G & 78 & 47 \\
8 & T80Del & 78 & $100^{*}$ \\
9 & G84C & 78 & 41 \\
10 & C42G & 76 & 53 \\
11 & G1A & 74 & 43 \\
12 & A110G & 65 & 48 \\
13 & A112T & 61 & 38 \\
14 & T19C & 59 & 37 \\
15 & T39G-C114Del & 57 & $0^{*}$ \\
16 & A151C & 56 & 52 \\
17 & G79C & 55 & 53 \\
18 & C89T & 53 & 49 \\
19 & G1T & 46 & 63 \\
20 & T39G-T101Del & 41 & $98^{*}$ \\
\hline
\end{tabular}

The sequences are ordered and numbered according to the number of identical copies of the sequence in the monomer dataset. The "Sequence" column indicates how each sequence differs from the consensus sequence of the C1 family, using standard notations. The "Number" column displays the number of identical copies of the sequence in the monomer dataset. The "Forward" column displays the percentage of reads obtained in the forward orientation (i.e. the orientation of our reference sequence)

Strong biases for read orientation are shown with an asterix $(*)$ 


\section{Characterization of alpha satellite diversity in the dimer dataset}

Among the 3,568 sequences recovered from the dimer dataset, 1,095 contained an intact XmnI restriction site approximately located in the middle, suggesting that the enzymatic digestion was not complete. The remaining 2,473 sequences, which did not possess the $\mathrm{XmnI}$ restriction site, were split using an alignment-based process (see Methods) and the resulting monomers were submitted to a length filter, giving a total of 2,408 associated left and right monomers. We focused first on these sequences and submitted them to the same process as described above. PCA showed the existence of two groups for both the left and right monomers, which could be discriminated using HCA (Fig. 3a and b). A comparison of the consensus sequences of each group revealed that the most abundant sequence set for both the left and right monomers belonged to the $\mathrm{C} 2$ group, while the two smaller sequence sets had consensus sequences that differed from each other and from the consensus of the $\mathrm{C} 1$ and $\mathrm{C} 2$ groups (Fig. 2). These two new groups of sequences, which represented $5 \%$ of the left or right monomers, will be from now on called $\mathrm{C} 3$ and $\mathrm{C} 4$ and shown in dark and light pink, respectively. We decided to build a phylogenetic tree with left and right monomers mixed together, using the described color code (Fig. 3c). This tree confirmed the existence of $\mathrm{C} 3$ and $\mathrm{C} 4$ as separate groups. Their respective average sequence identities were measured to be $86 \%$ and $83 \%$. We also checked that left and right monomers belonging to the $\mathrm{C} 2$ group could not be distinguished from each other on a phylogenetic tree (Additional file 1: Figure S2), or from the $\mathrm{C} 2$ sequences present in the monomer dataset. The comparison of the consensus sequences showed that the $\mathrm{C} 3$ and $\mathrm{C} 4$ groups differed much more from each other and from the $\mathrm{C} 1$ and $\mathrm{C} 2$ groups than $\mathrm{C} 1$ and $\mathrm{C} 2$ differed from each other. Interestingly, $\mathrm{C} 4$ was the only group with a consensus length of $171 \mathrm{bp}$ instead of $172 \mathrm{bp}$. A search for CENP-B and pJalpha boxes showed that most sequences within the $\mathrm{C} 4$ group contained a pJalpha box $(75 \%)$ while the CENP-B box was absent, like observed for the $\mathrm{C} 1$ and $\mathrm{C} 2$ groups. By contrast, neither the pJalpha box nor the CENP-B box was found in the sequences from the C3 group (Fig. 2).

The dimer dataset was also used to infer information regarding how monomers belonging to different groups associated with each other. All left and right monomers were assigned to one of the $\mathrm{C} 1$ to $\mathrm{C} 4$ groups (see Methods). Additional file 2: Table S2 reports the results of these assignments as well as associations between left and right monomers, distinguishing dimers that contained the XmnI site (X dataset) and those where the $\mathrm{XmnI}$ site was absent (noX dataset). We noticed that sequences from the $\mathrm{C} 1$ group were absent from the noX dataset and were poorly represented in the $\mathrm{X}$ dataset. This result may appear unexpected as $82 \%$ of the sequences from the monomer dataset belonged to the $\mathrm{C} 1$ group. Two hypotheses may explain this observation: the high sequence identity within the $\mathrm{C} 1$ group may reduce both the likelihood of the inactivation of the XmnI digestion site through mutations and the sequencing efficiency of dimers (see above). A statistical analysis of the X dataset showed that left monomers from the $\mathrm{C} 1$ and $\mathrm{C} 2$ groups were preferentially associated to right monomers from the same group (Additional file 2: Table S2), which suggests that sequences from the $\mathrm{C} 1$ and the $\mathrm{C} 2$ groups are tandemly repeated in the Cercopithecus solatus genome. C2-

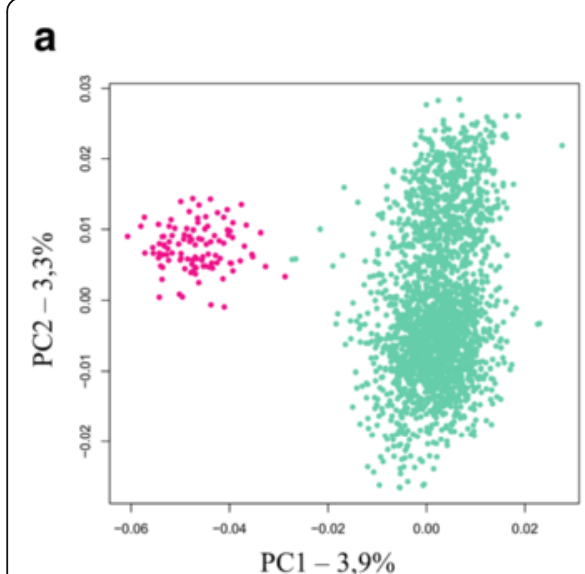

b

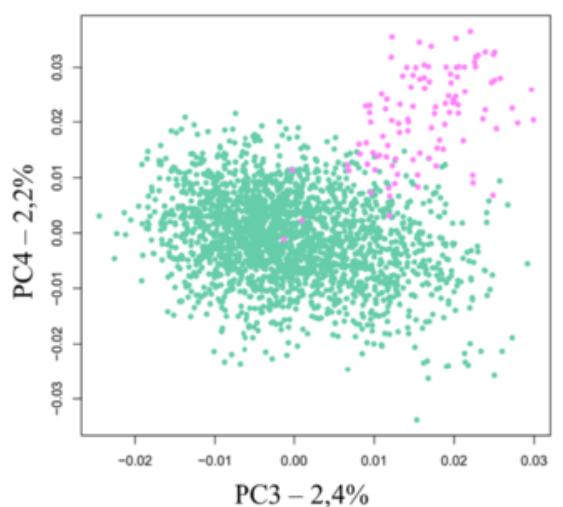

C

Fig. 3 Characterization of alpha satellite diversity in the dimer dataset. PCA projection of the normalized 5-mer frequency vectors are shown for (a) the left monomers and (b) the right monomers. Each point represents a sequence and has been colored according to its assignment to the C2 (pastel green), C3 (dark pink) or C4 (light pink) alpha satellite family based on hierarchical classification method. c Phylogenetic tree (Neighbor-joining method, K2P model, 100 bootstraps) for 250 randomly selected left monomers and 250 randomly selected right monomers. The color code matches the one described for (a) and (b). Bootstrap values for the branches leading to C3 and C4 are 87 and 55, respectively 
C2 associations were also found to predominate within the noX dataset. Interestingly, left monomers from the C3 group were preferentially associated to right monomers from the $\mathrm{C} 4$ group, suggesting the existence of a higher order organization with repeats containing at least two monomers belonging to different groups.

\section{Genomic distribution of alpha satellite families on Cercopithecus solatus chromosomes}

We were next interested in studying the genomic distribution of the four groups of sequences identified above. Short oligonucleotide probes have been shown to be more efficient at distinguishing alpha satellite sequences that differ by very few nucleotides compared with classical probes obtained by random priming or nick translation [42, 43]. We chose to use synthetic 18-mer oligonucleotides carrying locked nucleic acid (LNA) modifications at one out of two positions and capable of forming at least $7 \mathrm{GC}$ base pairs, as previous work had demonstrated their interest for the detection of alpha satellite sequences [44]. An in silico probe selection process was implemented in order to identify among the most common 18-mer sequences within a group (found in more than $20 \%$ of the monomers) those that were specific for this group (found in less than 3\% of the monomers of other groups). As we expected that oligonucleotide probes may still hybridize in the presence of one mismatch, we calculated the expected binding frequencies when one mismatch was present and applied the same selection criteria once again. Additional file 1: Figure S3 reports the sequences that best fitted with our requirements, albeit not completely. Due to the high sequence similarity between sequences within the $\mathrm{C} 1$ and $\mathrm{C} 2$ groups, probes had to distinguish sequences that differ mainly by only two nucleotides or even a single one (Additional file 1: Figure S3). The two sets of probes selected to target the $\mathrm{C} 1$ and $\mathrm{C} 2$ groups were therefore designed so that they would compete with each other if used simultaneously. The detection systems (fluorophores or haptens) were chosen in order to allow various combinations of probes to be tested together.

A first series of FISH experiments on Cercopithecus solatus metaphase spreads was performed using probes $\mathrm{Cla}$ and $\mathrm{C} 2 \mathrm{a}$ or $\mathrm{C} 1 \mathrm{~b}$ and $\mathrm{C} 2 \mathrm{~b}$ simultaneously. Probes targeting the $\mathrm{C} 1$ group produced intense signals at the centromere (primary constriction) of all chromosomes except a single one (Fig. 4a and c), while probes targeting the $\mathrm{C} 2$ group provided signals that are located in the pericentromeric

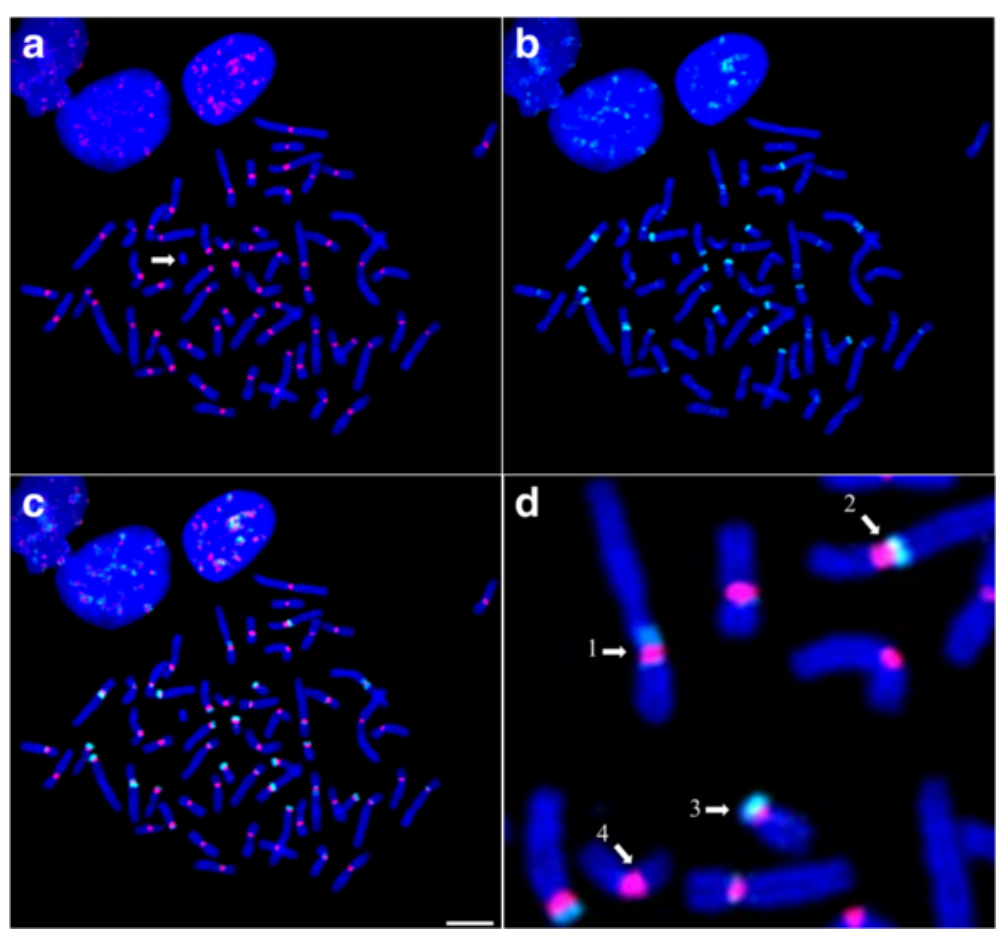

Fig. 4 FISH analysis of the $\mathrm{C} 1$ and $\mathrm{C} 2$ alpha satellite families characterized in the monomer dataset. Probes C1b and C2b are hybridized simultaneously to Cercopithecus solatus chromosomes, which are colored in blue. a Hybridization of probe C1b is shown in red. The arrow points to a single unlabeled chromosome. $\mathbf{b}$ Hybridization of probe $\mathrm{C} 2 \mathrm{~b}$ is shown in green. $\mathbf{c}$ Combined signals from (a) and (b). (d) Focus on image (c) showing in details the different types of distribution of the $\mathrm{C} 2 \mathrm{~b}$ signals relatively to $\mathrm{C} 1 \mathrm{~b}$. 1: C2b labels both pericentromeric regions, 2: C2b labels one pericentromeric region toward the long arm, 3: C2b labels one pericentromeric region toward the short arm of an acrocentric chromosome, 4: no C2b signal can be observed on this chromosome. Scale bar $=10 \mu \mathrm{m}$ 
regions (around the primary constriction) of several chromosomes pairs with different labeling patterns (Fig. 4b and $\mathrm{c}$ and Additional file 1: Figure S4a). Some chromosomes were extensively labeled by $\mathrm{C} 2$ probes on both sides of the centromere, others seem to be labeled at only one side, and others seemed to display no signal (see arrows on Fig. 4d). Stronger signals were observed on the acrocentric chromosome short arms (Additional file 1: Figure S4a). When these probes were used alone, each $\mathrm{C} 1 \mathrm{a}$ or $\mathrm{C} 2 \mathrm{a}$ probe produced a labeling pattern similar to what was observed in the presence of the other. On the contrary, each $\mathrm{C} 1 \mathrm{~b}$ or $\mathrm{C} 2 \mathrm{~b}$ probe used alone labeled regions that are larger than in the absence of the other. These experiments suggest that our probes may hybridize to sequences that differ by a single nucleotide (i.e. $\mathrm{C} 1 \mathrm{~b}$ binds to sequences from the $\mathrm{C} 2$ group and $\mathrm{C} 2 \mathrm{~b}$ binds to sequences from the $\mathrm{C} 1$ group) but that this binding is inhibited in the presence of an adequate competitor probe. In addition, when target sequences differ by at least two nucleotides, a specific detection is achieved in the absence of competitor.

Additional experiments showed that in presence of competitors, the signal produced by $\mathrm{Cla}$ overlapped with the signal produced by $\mathrm{C} 1 \mathrm{~b}$ and the signal produced by $\mathrm{C} 2 \mathrm{a}$ almost perfectly overlapped with the one produced by $\mathrm{C} 2 \mathrm{~b}$ (Additional file 1: Figure S5). This observation supports the idea that the labeling patterns observed with the chosen oligonucleotide probes reflect the distribution of the sequence groups identified by sequence analysis. Moreover, the absence of overlap between signals provided by probes targeting sequences from the $\mathrm{C} 1$ and $\mathrm{C} 2$ groups suggests that monomers within each group are clustered together and do not mix with each other. Combined with the arguments described above that are in favor of a tandem organization of monomers for both the $\mathrm{C} 1$ and $\mathrm{C} 2$ groups, these features support the fact that the $\mathrm{C} 1$ and $\mathrm{C} 2$ groups of sequences represent distinct families of alpha satellite DNA that display a monomeric organization in the genome of Cercopithecus solatus.
Further experiments were performed with probes targeting the $\mathrm{C} 3$ and $\mathrm{C} 4$ groups. All $\mathrm{C} 3$ and $\mathrm{C} 4$ probes provided identical labeling patterns, with a strong signal located on a single chromosome, as well as very weak pericentromeric signals on some other chromosomes (Fig. 5 and Additional file 1: Figure S5). The chromosome labeled by the $\mathrm{C} 3$ and $\mathrm{C} 4$ probes, which is in fact the chromosome that was not labeled by the probes targeting $\mathrm{C} 1$, was also identified to be the Y chromosome by cytogenetic experiments (Additional file 1: Figure S4b). The colocalization of probes targeting the $\mathrm{C} 3$ and $\mathrm{C} 4$ groups and the absence of overlap with probes targeting the $\mathrm{C} 1$ or $\mathrm{C} 2$ group is consistent with the sequence analysis described above. These results taken together suggest thus that sequences belonging to the $\mathrm{C} 3$ and $\mathrm{C} 4$ groups represent additional families of alpha satellite DNA that display a higher order organization within the genome of Cercopithecus solatus. As a further control of the consistency between the results from FISH experiments and sequence analysis, we showed that a 13-mer LNA probe that was designed to target the four $\mathrm{C} 1$ to $\mathrm{C} 4$ groups of sequences (called $\mathrm{Cx}$ ) provided signals that overlapped with the combined signals of probes targeting each group, i.e. was able to label all chromosomes within the centromeric and pericentromeric regions (Additional file 1: Figure S6).

We were also interested in studying the chromosomal distribution of some of the repeated sequences found in high copy number in the monomer dataset. The results of our previously described FISH experiments suggest that the specific detection of single nucleotide variations may be difficult to achieve using individual probes but that using several probes in competition may provide the possibility to achieve the required level of specificity. Therefore, we designed new oligonucleotide probes targeting a common region, aiming at distinguishing three different highly repeated sequences with single nucleotide variations (Fig. 6, see Methods). When all probes were used in combination, probes targeting sequence 4. (T39G

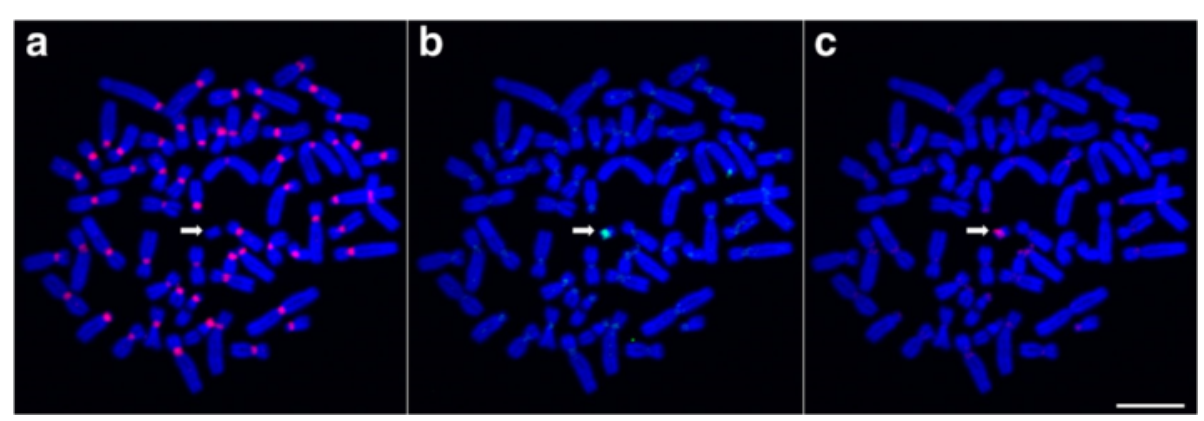

Fig. 5 FISH analysis of the C3 and C4 alpha satellite families characterized in the dimer dataset. Probes C1a, C3a and C4a are hybridized simultaneously to Cercopithecus solatus chromosomes, which are colored in blue. a Hybridization of probe Cla is shown in red. A single chromosome (shown with an arrow) is not labeled. $\mathbf{b}$ Hybridization of probe C3a is shown in green. $\mathbf{c}$ Hybridization of probe C4a is shown in red. The pericentromeric regions of several chromosomes are sparingly labeled by C3a and C4a. Scale bar $=10 \mu \mathrm{m}$ 


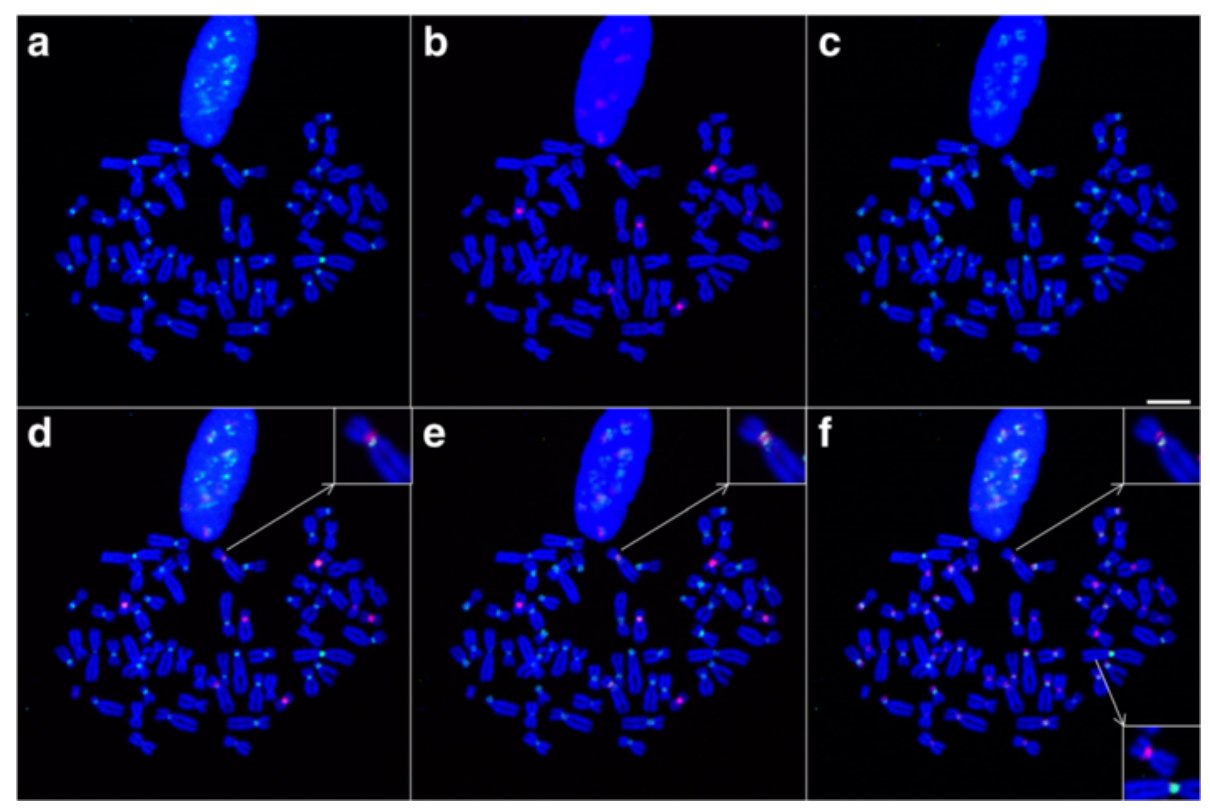

Fig. 6 FISH analysis of the chromosomal distribution of three sequences found in high copy number. Probes T39G, A40C and C42G and the competitor oligonucleotide TACco (complementary to the C1 consensus) are hybridized simultaneously to Cercopithecus solatus chromosomes, which are colored in blue. a Hybridization of probe T39G is shown in green. $\mathbf{b}$ Hybridization of probe A40C is shown in red. Eight chromosomes are labeled. c Hybridization of probe C42G is shown in green. $\mathbf{d}$ Combined signals from (a) and (b). e Combined signals from (b) and (c). f Combined signals from (a) and (c) with probe T39G shown in green and probe C42G shown in red. Upper inset in (d), (e) and (f) shows one chromosome where signals from the 3 probes do not overlap. Lower inset in (f) shows two chromosomes, one being labeled by probe T39G and the other by probe A40C. Scale bar $=10 \mu \mathrm{m}$

variation) and sequence 10 (C42G variation) seemed to label all chromosomes, albeit with non-overlapping patterns (see for example insets in Fig. 6f), while probe targeting sequence 5 (A40C variation) was clearly shown to produce a signal on only 8 chromosomes.

\section{Comparison of Cercopithecus solatus alpha satellite families with known primate families}

The sequence families defined above may provide information regarding the evolutionary history of alpha satellite DNA in Primates. We were therefore interested in investigating phylogenetic relationships between these families and alpha satellite sequences that were previously described for other primate species. Interestingly, the first alpha satellite consensus sequence ever described, which was obtained for the cercopithecini Chlorocebus aethiops [45], was exactly the same as the consensus sequence of our C1 family, which is also the most abundant repeated sequence in our dataset. This identity suggests the conservation of the C1 family between Cercopithecini species. Although very few sequences were available, a tentative classification was previously proposed for alpha satellite DNA present in Old and New World monkeys, involving five families termed S1 to S5 [17]. We built a phylogenetic tree containing 50 sequences randomly selected within each of our $\mathrm{C} 1$ to $\mathrm{C} 4$ families and several sequences representative for S1, S2, S4 and S5 (Fig. 7a, see Methods). The S1 sequences obtained from Chlorocebus aethiops were intermingled in this tree with our $\mathrm{C} 1$ and $\mathrm{C} 2$ sequences. Other sequences classified in S1 but obtained from other species were dispersed in other parts of the graph, suggesting that the proposed S1 family was not relevant. There was also no clear proximity of each one of the $\mathrm{C} 1$ to $\mathrm{C} 4$ family with sequences belonging to the so-called S2, S4 or S5 family. The phylogenetic tree showed on the contrary that sequences from macaque (identified as S1 or S2) may form a sister group of the C4 family whereas the only available baboon sequence (identified as S1) was close to the C3 family. None of the sequences from macaque or baboon resembled those from our $\mathrm{C} 1$ or $\mathrm{C} 2$ family. All these results suggest that, contrary to S4 and S5, S1 and S2 do not correspond to alpha satellite families. We also built a phylogenetic tree involving our C1-C4 families and seven families (termed M1, R1-2, V1, and $\mathrm{H} 1$ to $\mathrm{H} 4$ ) that were previously identified in human pericentromeric regions, some of them being reported as similar to sequences found in other primates [19] (Fig. 7b). The tree suggests that alpha satellite families found in $\mathrm{Cer}$ copithecus solatus have an evolutionary history that is largely independent from that of the alpha satellite families found in human pericentromeric regions.

\section{Discussion}

Despite the recent generalization of high-throughput sequencing, application of these new technologies to the 


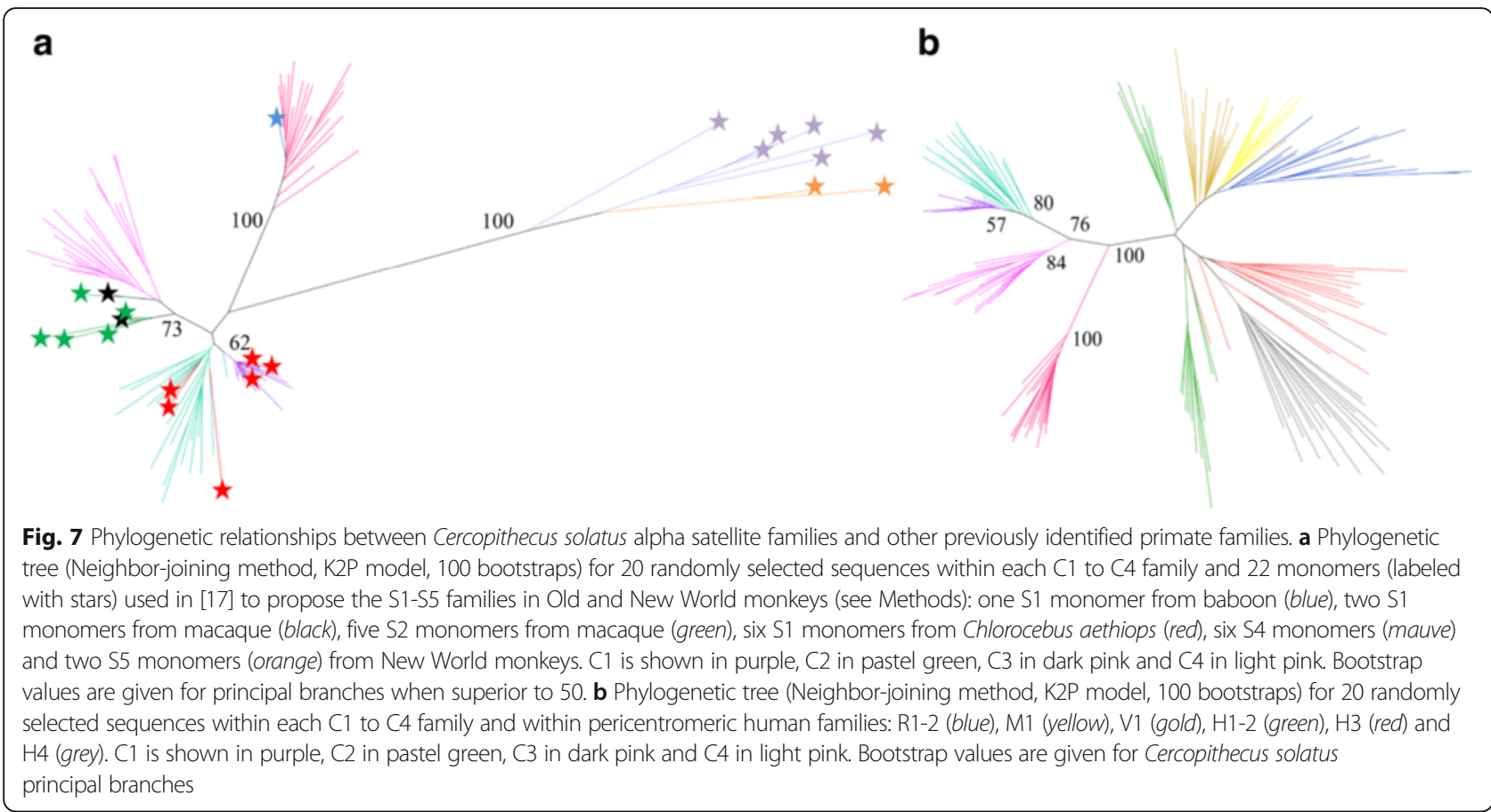

study of repeated DNA remains scarce [46, 47]. Here, we present an original experimental and computational framework for studying repeated DNA. We have focused on a single Cercopithecini species where the diversity and organization of alpha satellite DNA are described in details. Our approach relies on sequencing of gel purified alpha satellite monomers and dimers obtained by restriction enzyme digestion of genomic DNA, followed by sequence analysis and FISH experiments with carefully designed probes.

We detected four alpha satellite families, called $\mathrm{C} 1$ to $\mathrm{C} 4$, in the Cercopithecus solatus genome. Additional families may have been missed by our approach, for example because they would not contain restriction sites for XmnI. Although some technical issue had drastically reduced the number of available amount of sequences containing two monomers, the dimer dataset provided information about the structural organization of each family, showing that the $\mathrm{C} 1$ and $\mathrm{C} 2$ families adopt a monomeric organization, while $\mathrm{C} 3$ and $\mathrm{C} 4$ would associate into HORs. Our data do not allow concluding if the C3-C4 dimers are tandemly repeated or represent only a part of a longer HOR involving other monomers, but suggest that such structures, which have also been observed in New World monkey genomes [48], may be widespread in Primates. It had previously been reported, using a limited number of sequences, that alpha satellite sequences in Old World monkeys contained a pJalpha binding site and no CENP-B binding site [22, 49, 50]. Our data provide further support to this observation which holds true for three of the four newly identified families. The absence of any of these two binding sites in the C3 family represents an oddity but one should notice that as sequences from the C3 family are associated with sequences from the $\mathrm{C} 4$ family into a HOR organization, the pJalpha binding site remains present in the repeated motif. We detected several sequences in our dataset that were repeated identically a high number of times (up to several thousands). As our protocol does not contain any PCR amplification before capture of individual sequences on beads, the abundance of these sequences may reflect their natural abundance within the Cercopithecus solatus genome, provided one is able to identify potential artifacts resulting from sequencing errors among those sequences.

The high similarity between Cercopithecus solatus alpha satellite families, especially $\mathrm{C} 1$ and $\mathrm{C} 2$, the consensus of which differ at only a few nucleotide positions, required the implementation of a highly specific FISH detection to infer their chromosomal distribution. Our results emphasize the interest of short LNA-modified oligonucleotide probes that are here shown to be able to distinguish sequences that differ by only two nucleotides. It is even possible to distinguish a single nucleotide variation between two sequences by using two probes targeting each sequence variant simultaneously. In all our experiments, we cannot exclude the possibility that probes also hybridize to sequences that are not perfectly complementary, nor that some signals do not come from sequences that are present in the Cercopithecus solatus genome but not found in our datasets. Nevertheless, the absence of cross labeling between the probes targeting different families and the consistency of hybridization results with predictions inferred from sequence analysis 
support our probe design strategy and stands for the accuracy and the exhaustiveness of our description of the alpha satellite component of Cercopithecus solatus.

Our FISH experiments showed that the $\mathrm{C} 1$ family, which is the most conserved ( $95 \%$ mean sequence identity), displays a centromeric localization while the more divergent C2 family (85\% mean sequence identity) displays a pericentromeric localization. According to the age-gradient based model for centromere evolution [3,33], we may speculate that this pattern results from a peculiar evolutionary history where the $\mathrm{C} 2$ family, an old family of sequences, had occupied a centromeric position in an ancestor of Cercopithecus solatus. This family would then have been displaced towards pericentromeric regions following the amplification of more recent sequences from the $\mathrm{C} 1$ family at the centromere. Unequal crossing over at nearly identical repeats is thought indeed to lead to the homogenization of the core centromere, while mutations would only affect repeats outside of the core centromere [3, 19, 51-53]. An alternative but nonexclusive hypothesis would attribute distinct functional roles to both families, for example centromere function to $\mathrm{C} 1$ and cohesion of sister chromatids to $\mathrm{C} 2$, as it has been proposed for mouse minor and major satellite sequences, respectively [54]. Interestingly, acrocentric chromosome short arms display a very large amount of $\mathrm{C} 2$ sequences as revealed by intense FISH signals. This observation provides support to a previous hypothesis according to which acrocentric chromosomes may physically interact and exchange genetic material $[55,56]$. The fact that the $\mathrm{C} 3-\mathrm{C} 4$ dimers are found on the $\mathrm{Y}$ chromosome and are almost absent from other chromosomes may be explained by the fact that the $\mathrm{Y}$ chromosome is excluded from recombination events with non-homologous chromosomes, as was observed in mice [57]. Finally, the observation of the distribution of one of the highly repeated sequence variants on only 8 chromosomes supports the existence of local alpha satellite homogenization events in the Cercopithecus solatus genome.

Previous studies had considered alpha satellite DNA in Cercopithecini as poorly diversified [17]. Our results show that at least four alpha satellite families can be present in a single species, with complex chromosomal distribution and organizational patterns. Comparative studies including repetitive DNAs from different species have already been shown to provide new insights into genome and species evolution [58]. Our approach will permit not only to investigate the taxonomic distribution of alpha satellite families but also to study their organizational pattern, their chromosomal distribution as well as the existence of conserved highly repeated sequence variants. Phylogenetic analysis have demonstrated that the $\mathrm{C} 1$ to $\mathrm{C} 4$ families represent newly identified entities that do not correspond to previously proposed alpha satellite families. Although the available data are in favor of an apparent conservation of both the $\mathrm{C} 1$ and $\mathrm{C} 2$ families between Cercopithecus solatus and Chlorocebus aethiops, further studies will be required to better understand the dynamics of alpha satellite DNA in Old World monkeys and in other primates.

\section{Conclusions}

In summary, we have presented here a generally applicable strategy that provides, for a single species, a comprehensive description of alpha satellite sequence diversity and organization. Our approach, which is easy to implement and cost-effective, provides an opportunity to characterize satellite DNA in all species where a characteristic enzymatic ladder pattern can be obtained. Comparing different individuals and different species will provide new insights into the dynamics at which new satellite families or new highly repeated sequence variants appear during the course of evolution and transfer between chromosomes. The better description of the structure of heterochromatic regions also provides potential for enhancing the epigenetic characterization of these regions as well as understanding the regulatory functions of heterochromatin.

\section{Methods}

DNA collection and metaphase preparations

Fibroblast samples of Cercopithecus solatus (ID: 2012028, male sample, ethic permission $\mathrm{n}^{\circ}$ FR1207510445-I) from the Collection of cryopreserved living tissues and cells of vertebrates (RBCell collection, Muséum national d'Histoire naturelle, Paris) were used for DNA extraction and metaphase preparations. DNA was extracted using the Omega Biotek Tissue DNA Kit (Doraville, USA). Cell cultures and metaphase preparations were achieved according to [59].

\section{Alpha satellite DNA isolation and sequencing}

The Serial Cloner software (Serial Basics, serialbasics.free.fr) was used to perform in silico digestions of the Cercopithecini alpha satellite sequences registered as such in Genbank (Accession numbers: AM235889, AM235890, AM237210, AM237214, AM237213, AM237212, X04339, V00145, M26844 and AM237211), which contained both monomers and dimers. The restriction site of the XmnI restriction enzyme (GAANNNNTTC) was observed once in a great proportion of monomers and twice in almost all dimers. XmnI was then used to digest Cercopithecus solatus DNA in vitro. $10 \mu \mathrm{g}$ of Cercopithecus solatus genomic DNA were digested for $4 \mathrm{~h} 30 \mathrm{~min}$ at $37^{\circ} \mathrm{C}$ with 60 units of XmnI activity (New England Biolabs) in a total volume of $34 \mu \mathrm{L}$. The enzyme was inactivated for $20 \mathrm{~min}$ at $65^{\circ} \mathrm{C}$. The sample was loaded on a $1 \%$ agarose gel after addition of $6.8 \mu \mathrm{L}$ loading buffer ( $50 \%$ glycerol) and electrophoresis was performed in $0.5 \mathrm{X}$ Tris-borate-EDTA buffer, at room temperature for $2 \mathrm{~h} 45 \mathrm{~min}$ at $100 \mathrm{~V}$. The gel was briefly stained with ethidium bromide and then imaged by UV 
transillumination. Bands corresponding to alpha satellite monomers ( $170 \mathrm{bp})$ and dimers ( $340 \mathrm{bp})$ were cut and DNA was extracted from the gel with the Omega Biotek Gel extraction kit and resuspended in $100 \mu \mathrm{l}$ of elution buffer. About $220 \mathrm{ng}$ and $110 \mathrm{ng}$ were obtained for the $170 \mathrm{bp}$ and $340 \mathrm{bp}$ samples, respectively.

Sequencing was performed on a PGM sequencing platform (Ion Torrent technology) using the $400 \mathrm{bp}$ sequencing kit. Two libraries were generated using $50 \mathrm{ng}$ of both blunt digest pools and the Ion Plus Fragment Library Kit (4471252, Life Technologies) and tagged with Ion Xpress barcode adapters (4471250, Life Technologies). After purification (1.8X) with Ampure XP Beads (A63880, Agencourt Bioscience, Beverly, USA), the libraries were quantitated using a SsoAdvanced Sybr Green qPCR assay (Biorad, Hercules, USA) based on a custom $E$. coli reference library. After a dilution of each library down to $26 \mathrm{pM}, 0.22 \mathrm{fmol}$ for the $170 \mathrm{bp}$ library and $0.44 \mathrm{fmol}$ for the $340 \mathrm{bp}$ library were pooled as templates for the clonal amplification on Ion Sphere particles during the emulsion PCR, performed on a One Touch2 emPCR robot according to the Ion PGM Template OT2 400 Kit user guide (4479878, Life Technologies). The amplification products were loaded onto an Ion 316v2 chip (4483324, Life Technologies), and subsequently sequenced according to the Ion PGM Sequencing 400 Kit user guide (4482002, Life Technologies). After standard filtration of the raw reads (polyclonal and low quality removal), the Ion Torrent sequencing yielded 204,990 sequences for the $170 \mathrm{bp}$ pool and 353,683 sequences for the $340 \mathrm{bp}$ pool. They were deposited in the NIH Short Read Archive (SRA accession numbers SRX1595681 and SRX1595679).

\section{Alpha satellite sequence filtering}

All sequences with an average Phred score lower than 25 , a length outside the range $162-182 \mathrm{bp}$ for monomers and 324-364 bp for dimers, and sequences without the XmnI digested sites at the extremities $\left(5^{\prime}\right.$-NNTTC ... GAANN-3') were not considered for further analysis. Alpha satellite sequences were identified with a BLAST search against a reference alpha satellite sequence of Chlorocebus aethiops (AM23721) [60]. Using default BLAST parameters, all sequences exhibiting a hit longer than $80 \mathrm{bp}$ for monomers and $160 \mathrm{bp}$ for dimers were considered as alpha satellite sequences and conserved for the following analysis. All sequences were then reoriented if necessary in order to match the orientation of the reference alpha satellite sequence. The orientation information was preserved for investigations regarding reading biases.

Processing of dimeric sequences was performed as follows. When an XmnI site was present in the middle of these sequences, it was used for separating both monomers, providing the so-called left and right monomers located on the $5^{\prime}$ side and on the $3^{\prime}$ side of the sequence, respectively.
Dimers that did not contain any XmnI site in the middle were aligned against a synthetic sequence formed by two consecutive copies of the reference sequence using the Needleman-Wunsch algorithm [61] to identify the monomer limits and split them into left and right monomers according to the same rule as described above. All pairs with at least one monomer outside the 162-182 bp range were discarded. Pairing information was conserved to study association between left and right monomers.

\section{Alpha satellite sequence characterization}

Monomeric sequences were compared using their 5-mer composition in order to identify putative alpha satellite groups without direct alignment. For each set of monomers, the 5-mer frequency table was analyzed using a principal component analysis (PCA) to reduce the space complexity and enable data visualization on the first factorial planes. Sequences were classified into groups by using a hierarchical clustering method (HCA) based on the Ward criterion [62] applied to the Euclidean distances calculated from the 100 first principal components of the PCA. Because of the size of the monomer dataset, direct classification of the sequences using HCA was not possible. Instead, HCA was applied on 2,500 randomly selected sequences which were used to train a linear discriminant model. This model has been finally used to classify all the other monomers. The dimer dataset was analyzed in two different ways: 1) monomers extracted from dimers without XmnI sites were classified by using an HCA based on a PCA, 2) monomers extracted from dimers with a XmnI site have been classified by using a LDA trained to recognize the C1-C4 groups.

Because of the size of the datasets, the phylogenetic trees, the consensus sequences and the sequence distance analysis were conducted with different subsets of randomly selected sequences, using a homemade python script. The selected sequences were aligned using MUSCLE [63] and analyzed with SeaView [64]. The phylogenetic trees were built by using the Neighbor joining algorithm and the Kimura 2-parameters distance. Reliability of nodes was assessed using 100 bootstrap iterations. The relatively low bootstrap values observed in the trees can be explained by a limited number of family specific sites, i.e., the informative sites, into the alignments. Nevertheless, the same clustering of the families and the same relationship between these families have been observed with all the trees generated with different randomly selected sequences.

CENP-B and pJalpha boxes were searched with the patterns TTCGTTGGAARCGGGA and TTCCTTTTYCACCRTAG respectively [40] by using the program Fuzznuc [65] and allowing 2 mismatches. All statistical analyses were conducted with R [66]. Our R scripts and other programs are available upon request. 
The S1-S5 monomers used in Fig. 7a have been isolated from the sequences described in [17]. All these monomers have been extracted by using the homologous position of the XmnI digestion site as a starting point (XmnI phase) in order to be aligned with the monomers of Cercopithecus solatus. Unfortunately, no full length S3 monomer was available in this phase. To obtain the Genbank accession numbers and the alignment of the used S1-S5 monomers, see [17] and Additional file 3: Text S1. Human monomers from old and ancient families (M1, R1-2, V1, H1-H4) used in Fig. 7b have been isolated from the human $\mathrm{Xp}$ chromosome sequence (Genbank ID NT_011630) by using the homologous position of the XmnI digestion site as a starting point. Monomers have been assigned to a family according to their location along the sequence and the annotations provided in [19] (see Additional file 4: Text S2 for alignment).

\section{Oligonucleotide probes}

Short oligonucleotide probes (18 nucleotides in length) were designed in order to target specifically the different alpha satellite families identified in Cercopithecus solatus, by systematic prediction of binding frequencies based on the sequencing results. In some instances, when the 18mer sequence did not allow forming at least $7 \mathrm{GC}$ bp upon hybridization to the complementary strand, length was increased to 19. Sequences and binding frequencies are available in Additional file 1: Figure S3, which also provides details about the positions of locked nucleic acid (LNA) modifications in the probes. These positions were selected based on previous experience in order to achieve a good binding affinity and specificity [44]. When possible, we selected probes that were perfectly complementary to more than $20 \%$ of the sequences from the target group and to less than $3 \%$ of the sequences from the other groups. Additional file 1: Figure S3 also provides the expected binding frequencies if hybridization is possible despite the presence of one mismatch between the probe and its target. To target three sequences found in high copy number in the monomer dataset, we designed four LNAmodified probes (LNA are written in lower case and classic nucleotides are written in upper case): probe T39G (5'TgTtCtGtTCaTtCaTcTc3', 5'AlexaFluor488), probe A40C (5' 'TgTtCtGtGAaTtCaTcTc3', 3' Digoxygenin), probe C42G (5'TgTtCtCtTAaTtCaTcTc3', 3'Biotin) and probe TACco (5' TgTtCtGtTAaTtCaTcTc3') which is complementary to the $\mathrm{C} 1$ consensus sequence. LNA-modified probes were purchased from Eurogentec (Seraing, Belgium).

\section{FISH experiments}

FISH were performed on metaphase chromosome preparations. Hybridization solutions were prepared by diluting the oligonucleotide probes to a final concentration of $0.1 \mu \mathrm{M}$ in a hybridization solution consisting of $2 \mathrm{X}$ SSC pH 6.3, 50\% deionized formamide, $1 \mathrm{X}$ Denhardt solution, $10 \%$ dextran sulfate, and $0.1 \%$ SDS. $20 \mu \mathrm{L}$ of the hybridization solution were deposited on each slide and covered with a coverslip. The slides were then heated for $3 \mathrm{~min}$ at $70^{\circ} \mathrm{C}$ and hybridized for $1 \mathrm{~h}$ at $37^{\circ} \mathrm{C}$ in a Thermobrite apparatus (Leica Biosystems). Then, each slide was washed twice in $2 \mathrm{X} \mathrm{SSC}$ at $63^{\circ} \mathrm{C}$. Preparations were then incubated in blocking solution (4\% bovine serum albumin (BSA), 1X PBS, $0.05 \%$ Tween 20) for $30 \mathrm{~min}$ at $37^{\circ} \mathrm{C}$ to reduce nonspecific binding. Then, depending on the combination of probes, the following antibodies were used for subsequent revelations: Alexa 488-conjugated streptavidin (1:200; Life Technologies, Foster City, USA), Cy5-conjugated streptavidin (1:200; Caltag Laboratories, Burlingame, USA), FITC-conjugated sheep antidigoxigenin (1:200; Roche, Lewes, UK), and Rhodamineconjugated sheep anti-digoxigenin (1:200; Roche). All antibodies were diluted in blocking solution containing $1 \mathrm{X}$ PBS, $0.05 \%$ Tween 20, and 4\% BSA. Antibody incubation lasted for $30 \mathrm{~min}$ at $37^{\circ} \mathrm{C}$. All washings were performed in 2X SSC, $0.05 \%$ Tween 20. Chromosomes were counterstained with DAPI (4',6-diamidino-2-phenylindole) by pipetting $40 \mu \mathrm{L}$ of a $5 \mu \mathrm{g} / \mathrm{mL}$ solution onto the slides, incubating for $5 \mathrm{~min}$ and then briefly washing in 1X PBS. Slides were mounted by adding a drop of Vectashield Antifade Mounting Medium (Vector Laboratories, Burlingame, USA) and covering with a coverslip.

\section{Image acquisition and analysis}

Metaphases were imaged using an Axio Observer Z1 epifluorescent inverted microscope (Zeiss) coupled to an ORCA R2 cooled CDD camera (Hamamatsu). The Axio Observer Z1 was equipped with a Plan-Apochromat 63x 1.4 NA oil-immersion objective and the following filters set: 49 shift free for DAPI (G365 / FT395 / BP445/50), $38 \mathrm{HE}$ shift free for FITC/Alexa488 (BP470/40 / FT495 / BP525/50), homemade sets for Rhodamine (BP546/10 / FF555 / BP 583/22) and for Cy5 (BP643/20 / FF660 / BP684/24). The light source was LED illumination (wavelengths: $365 \mathrm{~nm}, 470 \mathrm{~nm}$ or $625 \mathrm{~nm}$ ) except for Rhodamine, for which a metal halide lamp HXP120 was preferred. Immersion oil of refractive index 1.518 at $23^{\circ}$ $\mathrm{C}$ was used. Color-combined images were reconstructed using ImageJ [67]. At least ten metaphases were visualized for each experiment, which all confirmed the described patterns.

\section{Additional files}

Additional file 1: Figure S1. Migration profiles of Cercopithecus solatus genomic DNA digested with Xmnl. Figure S2. Phylogenetic tree for left and right alpha satellite monomers from the dimer dataset. Figure S3. LNAmodified probes used to target the $\mathrm{C} 1$ to $\mathrm{C} 4$ alpha satellite families on 
Cercopithecus solatus chromosomes. Figure S4. Distribution pattern of C2 and C3 alpha satellite families on Cercopithecus solatus chromosomes. Figure S5. Comparison of FISH signals from different probes targeting identical alpha satellite families. Figure $\mathbf{5 6}$. Comparison of the hybridization pattern of the $\mathrm{Cx}$ probe with those of probes targeting the $\mathrm{C} 1$ to $\mathrm{C} 4$ alpha satellite families. (DOCX $1508 \mathrm{~kb}$ )

Additional file 2: Table S1. Filtering steps from Cercopithecus solatus raw data to alpha satellite monomer and dimer datasets. Table S2. Alpha satellite family associations in Cercopithecus solatus dimer dataset. (DOCX $40 \mathrm{~kb}$ )

Additional file 3: Text S1. Alignment used to generate the phylogenetic tree from Fig. 7a. (TXT $28 \mathrm{~kb}$ )

Additional file 4: Text S2. Alignment used to generate the phylogenetic tree from Fig. 7b. (TXT $48 \mathrm{~kb}$ )

\section{Acknowledgments}

This manuscript is dedicated to the memory of our friend and colleague Florence Richard. We are grateful to Peggy Motsch, the ECOSOL project (ECOlogie de Cercopithecus SOLatus) and the Centre International de Recherches Médicales de Franceville for providing the Cercopithecus solatus samples to the RBCell collection (MNHN). We thank the Service de Systématique Moléculaire (UMS2700, MNHN), and especially Delphine Gey, Régis Debruyne and José Utge for the sequencing experiment. We are much obliged to AnneMarie and Bernard Dutrillaux for their advice in metaphase preparation and analysis as well as to François Loll for the technical support he provided regarding FISH experiments and image acquisition.

\section{Funding}

This work was supported by the Actions Thématiques Muséum "Génomique et Collections" and "Emergence".

\section{Availability of data and material}

The datasets supporting the conclusions of this article are available in the $\mathrm{NIH}$ Short Read Archive under SRX1595681 (http://www.ncbi.nlm.nih.gov/sra/ SRX1595681) and SRX1595679 (http://www.ncbi.nlm.nih.gov/sra/SRX1595679) IDs.

\section{Authors' contribution}

$C E, F R$, $L C$ and $L P$ conceived and designed the experiments. $L C, L P$ and $M G$ performed the experiments. LC, LP and CE analyzed the data. LP and CE contributed reagents/materials/analysis tools. LC, CE and LP contributed to the writing of the manuscript. All authors read and approved the final manuscript.

\section{Competing interests}

The authors declared that they have no competing interests.

\section{Consent for publication}

Not applicable.

\section{Ethics approval and consent to participate}

Not applicable.

\section{Author details}

'Département Régulations, Développement et Diversité Moléculaire, Structure et Instabilité des Génomes, INSERM U1154, CNRS UMR7196, Sorbonne Universités, Muséum national d'Histoire naturelle, Paris, France. 2Département Systématique et Evolution, Institut de Systématique, Evolution, Biodiversité, UMR 7205 MNHN, CNRS, UPMC, EPHE, Sorbonne Universités, Muséum national d'Histoire naturelle, Paris, France. ${ }^{3}$ Université Versailles St-Quentin, Montigny-le-Bretonneux, France.

\section{Received: 14 April 2016 Accepted: 2 November 2016}

Published online: 14 November 2016

\section{References}

1. Yunis JJ, Yasmineh WG. Heterochromatin, satellite DNA, and cell function. Structural DNA of eucaryotes may support and protect genes and aid in speciation. Science. 1971;174:1200-9.

2. Warburton PE, Willard HF. Genomic analysis of sequence variation in tandemly repeated DNA. Evidence for localized homogeneous sequence domains within arrays of alpha-satellite DNA. J Mol Biol. 1990;216(1):3-16.
3. Schueler MG, Higgins AW, Rudd MK, Gustashaw K, Willard HF. Genomic and genetic definition of a functional human centromere. Science. 2001. 294(October):109-15.

4. Plohl M, Luchetti A, Meštrović N, Mantovani B. Satellite DNAs between selfishness and functionality: Structure, genomics and evolution of tandem repeats in centromeric (hetero)chromatin. Gene. 2008;409:72-82.

5. Feliciello I, Akrap I, Ugarković D. Satellite DNA Modulates Gene Expression in the Beetle Tribolium castaneum after Heat Stress. PLOS Genet. 2015;11(8): e1005466. Available from: http://dx.plos.org/10.1371/journal.pgen.1005466.

6. She X, Horvath JE, Jiang Z, Liu G, Furey TS, Christ L, et al. The structure and evolution of centromeric transition regions within the human genome. Nature. 2004;430(7002):857-64.

7. Maio JJ. DNA strand reassociation and polyribonucleotide binding in the African green monkey, Cercopithecus aethiops. J Mol Biol. 1971:56(3):579-95.

8. Musich PR, Brown FL, Maio JJ. Highly repetitive component alpha and related alphoid DNAs in man and monkeys. Chromosoma. 1980;80:331-48.

9. Maio JJ, Brown FL, McKenna WG, Musich PR. Toward a molecular paleontology of primate genomes. II. The Kpnl families of alphoid DNAs. Chromosoma. 1981;83:127-44.

10. Alves $\mathrm{G}$, Seuanez HN, Fanning T. Alpha satellite DNA in neotropical primates (Platyrrhini). Chromosoma. 1994;103(4):262-7.

11. Willard HF. Evolution of alpha satellite. Curr Opin Genet Dev. 1991:1(4):509-14

12. Rudd MK, Wray GA, Willard HF. The evolutionary dynamics of alpha-satellite. Genome Res. 2006;16:88-96.

13. Rudd MK, Willard HF. Analysis of the centromeric regions of the human genome assembly. Trends Genet. 2004;20:529-33.

14. Miga $\mathrm{KH}$. Completing the human genome: the progress and challenge of satellite DNA assembly. Chromosom Res. 2015;421-26. Available from: http://link.springer.com/10.1007/s10577-015-9488-2.

15. Schueler MG, Sullivan BA. Structural and functional dynamics of human centromeric chromatin. Annu Rev Genomics Hum Genet. 2006:7:301-13.

16. Miga KH, Newton Y, Jain M, Altemose N, Willard HF, Kent WJ. Centromere reference models for human chromosomes $X$ and $Y$ satellite arrays. 2014;24(4):697-707.

17. Alexandrov I, Kazakov A, Tumeneva I, Shepelev V, Yurov Y. Alpha-satellite DNA of primates: old and new families. Chromosoma. 2001;110:253-66.

18. Alkan C, Ventura M, Archidiacono N, Rocchi M, Sahinalp SC, Eichler EE. Organization and evolution of primate centromeric DNA from wholegenome shotgun sequence data. PLoS Comput Biol. 2007:3(9):1807-18.

19. Shepelev VA, Alexandrov AA, Yurov YB, Alexandrov I A. The evolutionary origin of man can be traced in the layers of defunct ancestral alpha satellites flanking the active centromeres of human chromosomes. PLoS Genet. 2009;5(9). Available from: http://dx.doi.org/10.1371/journal.pgen. 1000641.

20. Catacchio CR, Ragone R, Chiatante G, Ventura M. Organization and evolution of Gorilla centromeric DNA from old strategies to new approaches. Sci Rep. 2015; 5:14189. Available from: http://www.nature.com/doifinder/10.1038/srep14189.

21. Willard HF, Waye JS. Hierarchical order in chromosome-specific human alpha satellite DNA. Trends Genet. 1987;3(7):192-19.

22. Alexandrov IA, Medvedev LI, Mashkova TD, Kisselev LL, Romanova LY, Yurov YB. Definition of a new alpha satellite suprachromosomal family characterized by monomeric organization. Nucleic Acids Res. 1993;21(9):2209-15.

23. Hayden KE. Human centromere genomics: Now it's personal. Chromosom Res. 2012;20(July):621-33.

24. Alexandrov IA, Mitkevich SP, Yurov YB. The phylogeny of human chromosome specific alpha satellites. Chromosoma. 1988;96:443-53.

25. Lee C, Wevrick R, Fisher RB, Ferguson-Smith MA, Lin CC. Human centromeric DNAs. Hum Genet. 1997;100:291-304.

26. Jorgensen AL, Jones C, Bostock CJ, Bak AL. Different subfamilies of alphoid repetitive DNA are present on the human and chimpanzee homologous chromosomes 21 and 22. EMBO J. 1987:6(6):1691-6.

27. Archidiacono N, Antonacci R, Finelli P, Lonoce A, Rocchi M. Comparative Mapping of Human Alphoid Sequences in Great Apes Using Fluorescence. Genomics. 1995;484:477-84.

28. Warburton PE, Haaf T, Gosden J, Lawson D, Willard HF. Characterization of a chromosome-specific chimpanzee alpha satellite subset: evolutionary relationship to subsets on human chromosomes. Genomics. 1996:33(2): 220-8.

29. Malik HS, Henikoff S Conflict begets complexity: The evolution of centromeres. Curr Opin Genet Dev. 2002;12:711-8. 
30. Warburton PE, Willard HF. Interhomologue sequence variation of alpha satellite DNA from human chromosome 17: evidence for concerted evolution along haplotypic lineages. J Mol Evol. 1995;41(6):1006-15.

31. Schindelhauer D, Schwarz T. Evidence for a fast, intrachromosomal. conversion mechanism from mapping of nucleotide variants within a homogeneous alpha-satellite DNA array. Genome Res. 2002;12:1815-26.

32. Roizès $G$. Human centromeric alphoid domains are periodically homogenized so that they vary substantially between homologues. Mechanism and implications for centromere functioning. Nucleic Acids Res. 2006;34(6):1912-24.

33. Schueler MG, Dunn JM, Bird CP, Ross MT, Viggiano L, Rocchi M, et al. Progressive proximal expansion of the primate $X$ chromosome centromere. Proc Natl Acad Sci U S A. 2005;102(30):10563-8.

34. Guschanski K, Krause J, Sawyer S, Valente LM, Bailey S, Finstermeier K, et al. Next-Generation Museomics Disentangles One of the Largest Primate Radiations. Syst Biol. 2013;62(4):539-54. Available from: http://sysbio. oxfordjournals.org/cgi/doi/10.1093/sysbio/syt018.

35. Mammal Species of the World: A Taxonomic and Geographic Reference (3rd ed). 2005. Wilson D, Reeder D. editors. Johns Hopkins University Press.

36. Madhani HD, Leadon SA, Smith CA, Hanawalt PC. a DNA in African green monkey cells is organized into extremely long tandem arrays. J Biol Chem. 1986;261:2314-8.

37. Fittler F. Analysis of the a-Satellite DNA from African Green Monkey Cells by Restriction Nucleases. Eur J Biochem. 1977;352:343-52.

38. Harrison JS, International C, Medicales DR. A new species of guenon (genus Cercopithecus) from Gabon. J Zool. 1984;1988:561-75.

39. Lee TN, Singer MF. Structural organization of alpha-satellite DNA in a single monkey chromosome. J Mol Biol. 1982;161:323-42.

40. Rosandić M, Paar V, Basar I, Glunčić M, Pavin N, Pilaš I. CENP-B box and pJa sequence distribution in human alpha satellite higher-order repeats (HOR). Chromosom Res. 2006;14:735-53.

41. Bragg LM, Stone G, Butler MK, Hugenholtz P, Tyson GW. Shining a Light on Dark Sequencing: Characterising Errors in Ion Torrent PGM Data. PLoS Comput Biol. 2013;9(4). Available from: http://dx.doi.org/10.1371/journal. pcbi.1003031.

42. O'Keefe CL, Matera AG. Alpha satellite DNA variant-specific oligoprobes differing by a single base can distinguish chromosome 15 homologs. Genome Res. 2000;10:1342-50.

43. Silahtaroglu A, Pfundheller $\mathrm{H}$, Koshkin A, Tommerup N. LNA-modified oligonucleotides are highly efficient as FISH probes. Cytogenet Genome Res. 2004;37:32-7.

44. Ollion J, Loll F, Cochennec J, Boudier T, Escudé C. Cell cycle-dependent positioning of individual centromeres in the interphase nucleus of human lymphoblastoid cell lines. Mol Biol Cell. 2015;26(13):2550-60.

45. Rosenberg $H$, Singer M, Rosenberg M. Highly reiterated sequences of SIMIANSIMIANSIMIANSIMIANSIMIAN. Science. 1978;200(April):394-402.

46. Rojo V, Martínez-Lage A, Giovannotti M, González-Tizón AM, Cerioni PN, Barucchi VC, et al. Evolutionary dynamics of two satellite DNA families in rock lizards of the genus Iberolacerta (Squamata, Lacertidae): different histories but common traits. Chromosom Res. 2015;23(3):441-61.

47. Ruiz-ruano FJ, López-león MD, Cabrero J, Camacho JPM. High-throughput analysis of the satellitome illuminates satellite DNA evolution. Sci Rep. 2016; 6. Available from: http://dx.doi.org/10.1038/srep28333.

48. Sujiwattanarat P, Thapana W, Srikulnath K, Hirai Y, Hirai H, Koga A. Higherorder repeat structure in alpha satellite DNA occurs in New World monkeys and is not confined to hominoids. Sci Rep. 2015;5:10315. Available from: http://www.nature.com/doifinder/10.1038/srep10315.

49. Goldberg IG, Sawhney H, Pluta AF, Warburton PE, Earnshaw WC. Surprising deficiency of CENP-B binding sites in African green monkey alpha-satellite DNA: implications for CENP-B function at centromeres. Mol Cell Biol. 1996;16(9):5156-68.

50. Yoda K, Nakamura T, Masumoto H, Suzuki N, Kitagawa K, Nakano M, et al. Centromere Protein B of African Green Monkey Cells : Gene Structure, Cellular Expression, and Centromeric Localization. Mol Cell Biol. 1996;16(9):5169-77.

51. Smith GP. Evolution of repeated DNA sequences by unequal crossover. Science. 1976;191:528-35.

52. Henikoff S. Near the edge of a chromosome's "black hole". Trends Genet. 2002;18(4):165-7.

53. Henikoff JG, Thakur J, Kasinathan S, Henikoff S. A unique chromatin complex occupies young a-satellite arrays of human centromeres. Sci Adv. 2015;1:e1400234.
54. Guenatri M, Bailly D, Maison C, Almouzni G. Mouse centric and pericentric satellite repeats form distinct functional heterochromatin. JCB. 2004;166(4): 493-505.

55. Choo KH, Earle E, Mcquillan C. A homologous subfamily of satellite III DNA on human chromosomes 14 and 22. Nucleic Acids Res. 1990;18(19):5641-8.

56. Warburton PE, Hasson D, Guillem F, Lescale C, Jin X, Abrusan G. Analysis of the largest tandemly repeated DNA families in the human genome. BMC Genomics. 2008;9:533.

57. Pertile MD, Graham AN, Choo KHA, Kalitsis P. Rapid evolution of mouse Y centromere repeat DNA belies recent sequence stability. Genome Res. 2009:19(12):2202-13.

58. Mravinac B, Plohl M. Parallelism in evolution of highly repetitive DNAs in sibling species. Mol Biol Evol. 2010;27(8):1857-67.

59. Moulin S, Gerbault-Seureau M, Dutrillaux B, Richard FA. Phylogenomics of African guenons. Chromosom Res. 2008;16(5):783-99.

60. Altschul SF, Gish W, Miller W, Myers EW, Lipman DJ. Basic Local Alignment Search Tool. J Mol Biol. 1990;215:403-10.

61. Needleman SB, Wunsch CD. A general method applicable to the search for similarities in the amino acid sequence of two proteins. J Mol Biol. 1970; 48(3):443-53. Available from: http://www.sciencedirect.com/science/article/ pii/0022283670900574.

62. Ward JH. Hierarchical grouping to optimize an objective function. J Am Stat Assoc. 1963;58(301):236-44.

63. Edgar RC. MUSCLE : a multiple sequence alignment method with reduced time and space complexity. BMC Bioinform. 2004;19:1-19.

64. Gouy M, Guindon S, Gascuel O. SeaView Version 4 : A Multiplatform Graphical User Interface for Sequence Alignment and Phylogenetic Tree Building. Mol Biol Evol. 2010;27(2):221-4.

65. Rice P. The European Molecular Biology Open Software Suite EMBOSS : The European Molecular Biology Open Software Suite. Trends Genet. 2000;16(6):2-3.

66. R Core Team. R a Language and Environment for Statistical Computing. https://www.R-project.org.

67. Abràmoff MD, Hospitals I, Magalhães PJ, Abràmoff M. Image Processing with ImageJ. J Biophotonics. 2004;11(7):36-42.

\section{Submit your next manuscript to BioMed Central and we will help you at every step:}

- We accept pre-submission inquiries

- Our selector tool helps you to find the most relevant journal

- We provide round the clock customer support

- Convenient online submission

- Thorough peer review

- Inclusion in PubMed and all major indexing services

- Maximum visibility for your research

Submit your manuscript at www.biomedcentral.com/submit
Biomed Central 